\title{
Identificando sintomas de depressão em postagens do Twitter em português do Brasil
}

\author{
Augusto R. Mendes, Rafael V. P. Passador, Helena M. Caseli \\ ${ }^{1}$ Universidade Federal de São Carlos (UFSCar) \\ Departamento de Computação - LALIC \\ Caixa Postal 676 - 13565-905 - São Carlos - SP - Brasil \\ augustorm@estudante.ufscar.br, rafaelpassador@estudante.ufscar.br
}

AVISO: Este documento contém conteúdo sensível, com exemplos de termos e descrição de sintomas de transtornos depressivos.

\begin{abstract}
Currently, depression is one of the most worrisome mental health issues. In Brazil, in 2019, 10.2\% of the adult population reported having been diagnosed with depression according to data from the National Health Survey. Identifying people with a possible depressive profile allows adequate monitoring by mental health professionals. In this sense, online social networks such as Twitter can be important allies. This article presents experiments carried out for the automatic classification of Twitter posts (not users) containing content that denotes some symptom of depression. Logistic regression showed the best results (average F1 equal to 57\%) among the investigated algorithms.
\end{abstract}

Resumo. A depressão é uma das questões de saúde mental mais preocupantes da atualidade. No Brasil, em 2019, 10,2\% da população adulta relatou ter sido diagnosticada com depressão segundo dados da Pesquisa Nacional de Saúde. Identificar pessoas com perfil possivelmente depressivo permite um acompanhamento adequado por parte dos profissionais de saúde mental. Nesse sentido, as redes sociais online, como o Twitter, podem ser importantes aliadas. Este artigo apresenta experimentos realizados para a classificação automática de postagens (e não usuários) do Twitter contendo conteúdo que denota algum sintoma de depressão. A classificação com regressão logística apresentou os melhores resultados ( $F 1$ média de 57\%) entre os algoritmos investigados.

\section{Introdução}

O tratamento de depressão pode ser considerado uma das mais importantes questões de saúde mental da atualidade. No Brasil, segundo dados da Pesquisa Nacional de Saúde realizada em 2019, 10,2\% dos adultos brasileiros mencionaram terem recebido diagnóstico de depressão por profissional de saúde mental ${ }^{1}$.

A depressão maior pode ser diagnosticada quando uma pessoa apresenta, por pelo menos duas semanas, cinco ou mais sintomas, sendo que pelo menos um dos sintomas é humor deprimido ou perda de interesse ou prazer; os outros sintomas compreendem: diminuição ou aumento de peso ou apetite; insônia ou hipersonia; agitação ou retardo psicomotor; fadiga ou perda de energia; sentimentos de inutilidade ou culpa; concentração

\footnotetext{
${ }^{1}$ Disponível em: https://www.ibge.gov.br/estatisticas/sociais/saude/ 9160-pesquisa-nacional-de-saude.html?edicao=29270\&t=resultados
} 
diminuída, ou indecisão e/ou pensamentos recorrentes de morte. Segundo o DSM-5 [American Psychiatric Association et al. 2014], esses sintomas devem causar sofrimento clinicamente significativo ou prejuízo no funcionamento social, profissional ou em outras áreas importantes da vida do indivíduo.

O rastreio de sintomas depressivos e de funcionalidade, podem ser realizados por instrumentos auto aplicáveis, ajudando a fornecer informações sobre o humor e a funcionalidade do indivíduo em um recorte de tempo. Para o rastreio de sintomas depressivos são usados mecanismos como a escala Beck Depression Inventory II (BDI-II) [BECK et al. 1961] e para a funcionalidade, a Escala de Avaliação de Incapacidade da Organização Mundial da Saúde 2.0 (WHODAS 2.0)².

Identificar pessoas com Perfil Possivelmente Depressivo (PPD) possibilita intervenções e acompanhamento por profissionais da área da saúde e é essencial para o direcionamento efetivo de recursos de saúde mental. Nesse sentido, as Redes Sociais Online (RSO) podem ser importantes aliadas. Estudos como os de [De Choudhury et al. 2013] apontam que RSO são ambientes mais naturais para identificação de PPD quando comparados com os instrumentos comumente usados na área de saúde.

$\mathrm{Na}$ língua inglesa, existem diversos exemplos bem-sucedidos de técnicas de Processamento de Linguagem Natural (PLN) aplicadas para saúde mental, como a previsão de diagnósticos clínicos de depressão de usuários do Facebook através de suas postagens pré-diagnóstico [Eichstaedt et al. 2018], a detecção de ideação suicida em postagens no Reddit [Ji et al. 2018] e Twitter [O’Dea et al. 2015] e a classificação de notas de suicídio genuínas e falsas [Pestian et al. 2012].

No caso da língua portuguesa, a literatura é consideravelmente mais escassa. Em [Duque et al. 2018], os autores relatam a classificação de sentenças depressivas no Twitter. Em [Santos et al. 2020], descreve-se a construção de um córpus que visa apoiar tanto o reconhecimento das questões de saúde mental em mídias sociais online, quanto a análise temporal dessas doenças, com a apresentação de resultados iniciais de classificação de textos abordando ambas as tarefas.

Nesse contexto, este artigo apresenta experimentos realizados para a classificação automática de postagens (e não usuários) com conteúdo que denota algum sintoma de depressão. Para tanto, o córpus de [Santos et al. 2020], que não está anotado com sintomas, foi anotado via um processo de rotulação fraca com base em uma versão traduzida do léxico de [Yazdavar et al. 2017].

As principais contribuições deste trabalho são: (i) primeiro trabalho de classificação automática de sintomas de depressão no português do Brasil, (ii) versão em português do léxico de [Yazdavar et al. 2017], (iii) versão do córpus de [Santos et al. 2020] anotada (via rotulação fraca) com sintomas de depressão, e (iv) classificador automático de sintomas de depressão com $F 1$ média de $57 \%$.

\section{Trabalhos relacionados}

$\mathrm{Na}$ literatura foram propostos diferentes métodos em relação ao escopo, plataformas analisadas, padrão-ouro (gold standard), engenharia de features e modelos usados. Em geral,

\footnotetext{
${ }^{2}$ Disponível em: https://apps.who.int/iris/bitstream/handle/10665/43974/ 9788562599514_por.pdf? sequence=19
} 
os trabalhos comprovam que é possível captar informações relevantes sobre a saúde mental dos usuários a partir do conteúdo textual publicado por eles nas mídias sociais.

Em [Mowery et al. 2016], os autores investigaram a prevalência dos sintomas associados ao transtorno depressivo maior ao longo do tempo, nos Estados Unidos, com base em dados do Twitter. Para tanto, desenvolveram classificadores automáticos para discernir se um tweet apresenta (ou não) evidência de depressão com base em diversas features binárias que indicam a presença ou ausência de: (1) n-gramas que representam termos relacionados a um sintoma, (2) etiquetas de part-of-speech, (3) emoticons que podem expressar visualmente algum sintoma depressivo, (4) idade/gênero do autor do tweet, características inferidas por meio da frequência de termos agregados em um léxico, (5) grau de subjetividade e polaridade da postagem, (6) traços de personalidade e (7) outras características extraídas do LIWC. O SVM foi o algoritmo que apresentou o melhor desempenho geral para a classificação automática com base em $F 1$, e obteve performance variável na detecção de sintomas individuais indo de $35 \%$ para a detecção do sintoma "humor deprimido" até $75 \%$ no sintoma "fadiga ou perda de energia".

Em [Ziwei e Chua 2019], utilizou-se uma aplicação web para classificação de postagens depressivas publicadas no Twitter, em inglês, por meio da análise de palavras potencialmente depressivas, neutras e não depressivas presentes nos léxicos de [Yazdavar et al. 2017]. Esse mesmo recurso (léxicos) é utilizado em outros trabalhos, como [Yadav et al. 2020], onde os autores propuseram uma abordagem para identificar sintomas depressivos utilizando BERT (Bidirectional Representation from Transformers) e aprendizado multitarefa (multitask learning). Para tanto, os autores coletaram e anotaram um córpus composto por 12.155 tweets de usuários depressivos, com 3.738 deles anotados com as 9 classes de sintomas da escala PHQ-9 (Patient Health Questionnaire-9) obtendo uma alta precisão $(96,91 \%)$ na detecção de postagens depressivas. A identificação dos sintomas, pelo método proposto, obteve um $F 1$ médio de $75 \%$ superando em pelo menos 2 pontos percentuais os métodos usados na comparação.

Em [Santos et al. 2020], os autores reiteram a busca por sinais de problemáticas relacionadas à saúde mental no Brasil. Para tanto, utilizaram um córpus composto por postagens de usuários do Twitter (detalhado na seção 3.2) que foram, então, divididas em: (i) postagens escritas antes do diagnóstico/tratamento e (ii) postagens escritas durante/depois do evento. Os autores utilizaram recursos linguístico-computacionais (LIWC, word embeddings), TF-IDF e algoritmos de AM (regressão logística, MLP). Os classificadores comparados foram: regressão logística com TF-IDF, multilayer perceptron com média de word embeddings e regressão logística com frequências de categorias do LIWC. Conclui-se que regressão logística com TF-IDF supera as alternativas (com F1 médio de $69 \%$ contra $59 \%$ alcançado pelo segundo melhor método, MLP com word embeddings).

Este artigo apresenta experimentos desenvolvidos com o córpus de [Santos et al. 2020] e uma versão traduzida do léxico de [Yazdavar et al. 2017] para a identificação automática dos 9 sintomas de depressão da PHQ-9. Todos esses recursos usados neste trabalho são apresentados na seção 3. 


\section{Recursos}

Esta seção descreve os recursos utilizados neste trabalho para identificar automaticamente os 9 sintomas de depressão da PHQ-9 ${ }^{3}$ [Spitzer et al. 1999]: (1) falta de interesse, (2) tristeza/humor depressivo, (3) desordem de sono, (4) falta de energia, (5) desordem alimentar, (6) baixa auto-estima, (7) problemas de concentração, (8) hiperatividade/baixa atividade e (9) pensamentos de suicídio.

\subsection{Lista semente de [Yazdavar et al. 2017] traduzida para o português}

Com a ajuda de um profissional de psicologia clínica, em [Yazdavar et al. 2017] foi produzida uma lista de termos relacionados a cada sintoma de depressão avaliado pelo PHQ9. Para tanto, os construtores da lista usaram a ferramenta Big Huge Thesaurus ${ }^{4}$ e o dicionário colaborativo de gírias Urban Dictionary ${ }^{5}$ para ajudar na busca por sinônimos. O léxico final contém mais de 1.620 entradas em inglês relacionadas à depressão. Além dos 9 sintomas da PHQ-9, os autores consideraram um décimo em seu léxico: (10) medicamentos relativos a depressão.

Como parte do presente trabalho, a lista original (em inglês) foi traduzida para o português por dois nativos do português, com bons conhecimentos em inglês e com auxílio do tradutor automático Google Translate ${ }^{6}$. O processo consistiu na entrada dos termos e subsequente avaliação dos resultados, levando em consideração o sintoma associado, bem como adequação com o vocabulário cotidiano. Nos casos em que o tradutor não forneceu saída adequada a partir da entrada fora de contexto, buscou-se traduzir uma sentença completa na qual a entrada era usada em um contexto relevante ao sintoma ${ }^{7}$.

Ao final deste processo, um conjunto de 1.213 seeds, em português, foi obtido. Diferenças no número de seeds em relação ao conjunto original têm duas causas. Primeiro, o conjunto continha expressões coloquiais (como "blothpick"), que nem sempre têm traduções óbvias para o português, foram descartadas. Outro fator para a diferença de quantidade é que várias entradas diferentes no inglês acabavam tendo a mesma tradução. Além disso, boa parte dos termos do léxico original têm gênero neutro (como "depressed"), resultando na geração de mais termos ao traduzir ("deprimido" e "deprimida", por exemplo).

A Tabela 1 traz exemplos de palavras e expressões associados a cada um dos sintomas, que no léxico original e no traduzido são identificados como "sinais" (signals).

\subsection{Córpus de [Santos et al. 2020]}

Em [Santos et al. 2020], os autores coletaram um córpus do Twitter que consiste em usuários que relataram terem sidos diagnosticados com transtornos de saúde mental por profissionais da saúde, ou que relataram terem iniciado os tratamentos para uma dessas

\footnotetext{
${ }^{3} \mathrm{O}$ Patient Health Questionnaire (PHQ-9) (https://images.app.goo.gl/ G6VoWfHL2Y4YVvLaA) é um questionário de 9 itens usado para diagnóstico de depressão, voltado para rápida aplicação por clínicos gerais.

${ }^{4}$ Disponível em: https: / / words . bighugelabs.com/

${ }^{5}$ Disponível em: https ://www.urbandictionary.com/

${ }^{6}$ Disponível em: https: / / translate.google.com/

${ }^{7}$ Por exemplo, o tradutor automático gerou a equivalente "blues" para a palavra "bluesy”, mas quando a mesma palavra foi empregada na sentença "I'm feeling bluesy" o tradutor foi capaz de gerar a tradução "Estou me sentindo triste".
} 
Tabela 1. Exemplos de entradas no léxico de sintomas traduzido para o português

\begin{tabular}{cllc}
\hline Sinal & Sintoma associado & Exemplo & $\mathbf{n}^{\circ}$ de termos \\
\hline 1 & falta de interesse & sem_graça, apatia, tédio & 178 \\
2 & tristeza/humor depressivo & melancólico,deprimente, abatido & 192 \\
3 & desordem de sono & insônia, sonolento, sem_dormir & 82 \\
4 & falta de energia & não_tenho_força, preguiça, cansado & 95 \\
5 & desordem alimentar & odeio_minhas_coxas, anorexia, barrigudo & 146 \\
6 & baixa auto-estima & não_mereço, desprezível, eu_me_odeio & 112 \\
7 & problemas de concentração & dispersa,distraído, desorientado & 96 \\
8 & hiperatividade/baixa atividade & ansiedade, agitado, inquieto & 81 \\
9 & pensamentos de suicídio & me_matar, merece_morrer, sono_eterno & 104 \\
10 & medicamentos & lítio, alprazolam, citalopram & 127 \\
\hline
\end{tabular}

condições. Para tanto, a coleta se baseou em termos relacionados à saúde mental (como depressão e ansiedade) e ao diagnóstico, tratamento, ou o uso de medicamentos antidepressivos.

Em seguida, todas as mensagens coletadas foram manualmente inspecionadas para filtrar aquelas que pareciam suficientemente genuínas. As 3.200 mensagens mais recentes dos autores foram, então, rotuladas apenas no nível do usuário. Para cada autor selecionado, as mensagens foram examinadas de modo a identificar o momento específico em que o diagnóstico ou tratamento foi iniciado, e para destacar o subconjunto de mensagens que foram publicados antes desse evento.

Criou-se também um grupo de controle constituído por usuários que manifestaram interesse em questões de saúde mental como (i) uma preocupação geral (por exemplo, promovendo a campanha de prevenção de suicídio "Setembro Amarelo"), (ii) uma preocupação em relação a uma pessoa particular que sofria de um problema de saúde mental (por exemplo, um amigo), ou (iii) por ser um estudante de psicologia com um interesse no tema da depressão. As mensagens obtidas em resposta a essas consultas foram inspecionadas manualmente para remover usuários diagnosticados ou tratados para problemas de saúde mental.

\section{Experimentos}

Essa seção descreve as etapas do experimento realizado para classificar automaticamente os 9 sintomas da PHQ-9 no córpus de [Santos et al. 2020].

\subsection{Rotulação fraca do córpus}

Com o intuito de realizar a identificação automática de sintomas de depressão adotouse a estratégia de rotulação fraca. ${ }^{8}$ Tal estratégia foi adotada porque não há disponível livremente um córpus anotado com tais sintomas para o português do Brasil, como havia no caso de [Yadav et al. 2020].

Assim, a partir do córpus de [Santos et al. 2020], no qual cada postagem está associada a uma de duas classes (positiva ou negativa) para o perfil depressivo, uma rotulação

\footnotetext{
${ }^{8} \mathrm{~A}$ rotulação fraca é uma técnica para rotulação automática de um córpus a partir de casamento de padrão de uma lista de termos com os textos a serem rotulados
} 
fraca foi aplicada para anotar os sintomas. Neste processo de rotulação fraca, os novos rótulos de uma dada postagem são atribuídos com base em dois fatores: (i) a classe atribuída para a tarefa de detecção de depressão, e (ii) a presença de palavras-chave associadas a um dado sintoma. Assim, apenas as postagens rotuladas como positivas (PPD) no córpus de [Santos et al. 2020] foram processadas para associar, via rotulação fraca, $\mathrm{a}(\mathrm{s})$ classe(s) do(s) sintoma(s) que contém. A Tabela 2 traz as quantidades de instâncias para cada sintoma.

Tabela 2. Quantidade de instâncias para cada sintoma

\begin{tabular}{l|c}
\hline Sintoma & \# instâncias \\
\hline 1 - falta de interesse & 5.052 \\
2 - tristeza/humor depressivo & 17.218 \\
3 - desordem de sono & 1.400 \\
4 - falta de energia & 4.854 \\
5 - desordem alimentar & 1.616 \\
6 - baixa auto-estima & 7.380 \\
7 - problemas de concentração & 2.162 \\
8 - hiperatividade/baixa atividade & 4.146 \\
9 - pensamentos de suicídio & 10.190 \\
\hline
\end{tabular}

\subsection{Pré-processamento do córpus}

Tratando-se de textos produzidos para mídias sociais, as postagens apresentam muitas abreviações, erros ortográficos, links e artefatos típicos de uma dada plataforma (por exemplo, menções no formato “@ username” no Twitter). Estas características constituem ruído e afetam negativamente a extração de features e posterior treinamento de modelos. Portanto, foi usada a ferramenta Enelvo [Bertaglia e Nunes 2016] para a normalização de abreviações (por exemplo, "vc" é normalizado para "você"), erros ortográficos e identificação de ruídos (por exemplo, urls são todas normalizadas para a forma "url"), que foram subsequentemente removidos.

\subsection{Extração de features}

As seguintes features foram utilizadas pelos algoritmos de aprendizado de máquina:

LIWC - Utilizando o LIWC para o português ${ }^{9}$, foi feita a contagem do número de termos, em cada postagem, que pertenciam às seguintes categorias: family, anx, sad, ingest, work, money, death, friend, health. Dessa forma, visou-se capturar informação a respeito de fatores sociais, econômicos e outros relacionados a sintomas como alimentação. Ao final deste processo, nove features binárias indicando presença (1) ou ausência (0) de termos em cada categoria foram geradas.

TF-IDF - Foi feita a extração da frequência de cada termo, em cada postagem, com contra-peso de acordo com a frequência de postagens como um todo (TF-IDF), com o intuito de capturar a temática e características de uma dada postagem que a diferencia das demais. Um vetor numérico contendo os valores de TF-IDF foi gerado neste processo.

\footnotetext{
${ }^{9}$ Disponível em: http://143.107.183.175:21380/portlex/index.php/pt/ projetos/liwe
} 
Polaridade - Neste trabalho, realizou-se o ajuste fino do modelo BERTimbau [Souza et al. 2020] com o córpus TweetsentBR [Brum e Nunes 2018], tendo como resultado um classificador de polaridade que atingiu $67 \%$ de precisão no treinamento. Este modelo foi, então, usado para determinar a polaridade de uma postagem como a polaridade majoritária das sentenças que a compõem. ${ }^{10}$ Uma feature categórica (-1 para negativa, 0 para neutra e 1 para positiva) foi gerada neste processo.

Embeddings - Utilizando as NILC-embeddings ${ }^{11}$, modelo CBOW de dimensão 100, foram calculados os vetores para cada palavra em uma dada postagem. A representação da postagem foi gerada como a média dos vetores de palavra. Além das embeddings pré-treinadas, foram usadas também embeddings treinadas a partir do córpus (por meio da biblioteca gensim ${ }^{12}$ ), com peso determinado pelo valor TF-IDF de cada palavra aplicado ao vetor da mesma. Assim, ao final deste processo, duas representações para a postagem foram usadas como features: uma baseada nas NILC-embeddings e outra baseada nas embeddings treinadas para o córpus deste trabalho.

As features extraídas foram, então, usadas pelos algoritmos SVM, regressão logística e MLP. O treinamento dos modelos foi realizado via scikit-learn ${ }^{13}$. Foi feita redução de dimensionalidade das features por meio de PCA, seguindo o seguinte critério: dos 50 componentes extraídos, foram incluídos todos aqueles que explicassem cumulativamente $90 \%$ de variância, sendo o restante descartados. Foi feita amostragem do córpus de forma que os conjuntos de treino e teste fossem balanceados.

\section{Resultados}

A Tabela 3 resume as configurações de melhor resultado, em termos de $F 1$, para cada sintoma. Esses valores foram obtidos considerando-se a validação cruzada (5-fold) no treinamento dos modelos a partir do conjuntos de instâncias (Tabela 2) para cada sintoma, com diferentes combinações de features. Estas configurações estão listadas na tabela no formato: Algoritmo usado + combinação de features entre parênteses (e.g: $\operatorname{LogReg}(\mathrm{TF}-$ IDF + LIWC).

A regressão logística (LogReg) foi o algoritmo de melhor desempenho na classificação de 6 das 9 classes de sintomas. As features TF-IDF e LIWC se destacaram, enquanto o impacto da polaridade do texto na performance é inconclusivo (como pode-se observar na Tabela 3). A comparação direta com os trabalhos relacionados não é indicada neste caso, uma vez que idioma e modo de anotação (rotulação fraca) adotados aqui diferem dos trabalhos da literatura. Contudo, vale mencionar que este trabalho estende [Santos et al. 2020] e corrobora sua constatação de que regressão logística com TF-IDF tem bom desempenho na análise de postagens com PPD.

É importante ressaltar que a estratégia de rotulação fraca apresenta limitações. Apesar de ela se basear na presença de diagnóstico prévio e na ocorrência de palavras e expressões associadas aos sintomas, não é possível garantir que a postagem em questão

\footnotetext{
${ }^{10}$ Em caso de empate, adotou-se a polaridade neutra.

${ }^{11}$ Disponível em: http://www.nilc.icmc.usp.br/embeddings

${ }^{12}$ Disponível em: https : / / radimrehurek.com/gensim/

${ }^{13}$ Disponível em: https://scikit-learn.org/
} 
Tabela 3. Melhores resultados de $F 1$ para cada sintoma

\begin{tabular}{l|c|l}
\hline Sintoma & Melhor valor de $F 1$ & Configuração do melhor resultado \\
\hline 1 - falta de interesse & $54,66 \%$ & LogReg (TF-IDF + LIWC + polaridade) \\
2 - tristeza/humor depressivo & $56,09 \%$ & LogReg (TF-IDF + LIWC + polaridade) \\
3 - desordem de sono & $56,47 \%$ & SVC (embeddings com peso) \\
4 - falta de energia & $58,59 \%$ & LogReg (TF-IDF + LIWC) \\
5 - desordem alimentar & $60,22 \%$ & LogReg (TF-IDF + LIWC + polaridade) \\
6 - baixa auto-estima & $55,35 \%$ & SVC (embeddings com peso) \\
7 - problemas de concentração & $55,83 \%$ & LogReg (TF-IDF + LIWC + polaridade) \\
8 - hiperatividade/baixa atividade & $58,11 \%$ & SVC (embeddings com peso) \\
9 - pensamentos de suicídio & $57,66 \%$ & LogReg (TF-IDF + LIWC) \\
\hline Média & $56,99 \%$ & - \\
\hline
\end{tabular}

relata mesmo o sintoma. A rotulação fraca é especialmente prejudicada pela presença de ambiguidade e pela subjetividade da tarefa, uma vez que nem toda postagem produzida por uma pessoa deprimida necessariamente evidencia sintoma de depressão.

Nesse ínterim, observa-se a incidência de falsos positivos e falsos negativos. Por exemplo, analisando as anotações feitas para o sintoma "desordem de sono" pelo SVC (embeddings com peso), as postagens classificadas que continham os termos "cansada", "preguiça" e "cansaço" apresentaram maiores ocorrências de falsos positivos.

\section{Conclusões e Trabalhos futuros}

Como discutido na seção 5, a rotulação fraca apresenta limitações especialmente no contexto de tarefas como a investigada neste artigo, na qual o conteúdo deve ser avaliado considerando critérios subjetivos e instrumentos da saúde mental. Assim sendo, um dos trabalhos futuros será realizar a anotação do córpus com o auxílio de especialistas em saúde mental, uma vez que esta parece ser a melhor estratégia dadas as características da tarefa proposta. Também como trabalho futuro, pretende-se submeter a lista semente traduzida para a revisão por especialistas em saúde mental e linguística, de forma a validar o recurso para a língua portuguesa, garantindo maior qualidade de tradução.

Mesmo com as presentes limitações, os experimentos conduzidos produziram modelos capazes de classificar sintomas com $F 1$ entre $55 \%$ e $60 \%$, indicando a viabilidade da tarefa. Trabalhos futuros buscarão investigar novos conjuntos de features e técnicas de classificação, como a utilização de modelos pré-treinados baseados em transformers [Yadav et al. 2020].

Todos os recursos e códigos produzidos nesta pesquisa estão disponíveis em https://github.com/LALIC-UFSCar/Amive-PLN

\section{Agradecimentos}

Agradecemos ao professor Ivandré Paraboni pela disponibilização do córpus utilizado neste trabalho. Agradecemos também o suporte financeiro das entidades de apoio e incentivo à pesquisa acadêmica: o Conselho Nacional de Desenvolvimento Científico e Tecnológico (CNPq) e a Fundação de Amparo a Pesquisa do Estado de São Paulo (FAPESP). Em especial as bolsas PIBIC e IC FAPESP (\#2021/02430-9) e o projeto Amive (Auxílio Regular FAPESP \#2020/05157-9) do qual esta pesquisa faz parte. 


\section{Referências}

American Psychiatric Association et al. (2014). DSM-5: Manual diagnóstico e estatístico de transtornos mentais. Artmed Editora.

BECK, A. T., WARD, C. H., MENDELSON, M., MOCK, J., e ERBAUGH, J. (1961). An Inventory for Measuring Depression. Archives of General Psychiatry, 4(6):561-571.

Bertaglia, T. F. C. e Nunes, M. d. G. V. (2016). Exploring word embeddings for unsupervised textual user-generated content normalization. In Proceedings of the 2 nd Workshop on Noisy User-generated Text (WNUT), pages 112-120.

Brum, H. e Nunes, M. d. G. V. (2018). Building a Sentiment Corpus of Tweets in Brazilian Portuguese. In chair), N. C. C., Choukri, K., Cieri, C., Declerck, T., Goggi, S., Hasida, K., Isahara, H., Maegaard, B., Mariani, J., Mazo, H., Moreno, A., Odijk, J., Piperidis, S., e Tokunaga, T., editors, Proceedings of the Eleventh International Conference on Language Resources and Evaluation (LREC 2018), Miyazaki, Japan. European Language Resources Association (ELRA).

De Choudhury, M., Counts, S., e Horvitz, E. (2013). Social media as a measurement tool of depression in populations. In Proceedings of the 5th Annual ACM Web Science Conference, WebSci '13, page 47-56, New York, NY, USA. Association for Computing Machinery.

Duque, J. W. G., Raymundo, A. L., e Neto, P. F. (2018). Uma aplicação de big data para classificação de sentenças depressivas do twitter. Revista H-TEC Humanidades e Tecnologia, 2(1):82-95.

Eichstaedt, J. C., Smith, R. J., Merchant, R. M., Ungar, L. H., Crutchley, P., PreoţiucPietro, D., Asch, D. A., e Schwartz, H. A. (2018). Facebook language predicts depression in medical records. volume 115, pages 11203-11208. National Academy of Sciences.

Ji, S., Yu, C., Fung, S.-f., Pan, S., e Long, G. (2018). Supervised learning for suicidal ideation detection in online user content. Complexity, 2018:1-10.

Mowery, D. L., Park, A., Bryan, C., e Conway, M. (2016). Towards automatically classifying depressive symptoms from Twitter data for population health. In Proceedings of the Workshop on Computational Modeling of People's Opinions, Personality, and Emotions in Social Media (PEOPLES), pages 182-191, Osaka, Japan. The COLING 2016 Organizing Committee.

O’Dea, B., Wan, S., Batterham, P. J., Calear, A. L., Paris, C., e Christensen, H. (2015). Detecting suicidality on twitter. Internet Interventions, 2(2):183-188.

Pestian, J., Matykiewicz, P., Linn-Gust, M., South, B., Uzuner, O., Wiebe, J., Cohen, K., Hurdle, J., e Brew, C. (2012). Sentiment analysis of suicide notes: A shared task. Biomedical informatics insights, 5:3-16.

Santos, W., Funabashi, A., e Paraboni, I. (2020). Searching brazilian twitter for signs of mental health issues. In Proceedings of The 12th Language Resources and Evaluation Conference, pages 6111-6117, Marseille, France. European Language Resources Association. 
Souza, F., Nogueira, R., e Lotufo, R. (2020). BERTimbau: pretrained BERT models for Brazilian Portuguese. In 9th Brazilian Conference on Intelligent Systems, BRACIS, Rio Grande do Sul, Brazil, October 20-23 (to appear).

Spitzer, R. L., Kroenke, K., Williams, J. B., Group, P. H. Q. P. C. S., Group, P. H. Q. P. C. S., et al. (1999). Validation and utility of a self-report version of prime-md: the phq primary care study. Jama, 282(18):1737-1744.

Yadav, S., Chauhan, J., Sain, J. P., Thirunarayan, K., Sheth, A., e Schumm, J. (2020). Identifying depressive symptoms from tweets: Figurative language enabled multitask learning framework. In Proceedings of the 28th International Conference on Computational Linguistics, pages 696-709, Barcelona, Spain (Online). International Committee on Computational Linguistics.

Yazdavar, A. H., Al-Olimat, H. S., Ebrahimi, M., Bajaj, G., Banerjee, T., Thirunarayan, K., Pathak, J., e Sheth, A. (2017). Semi-supervised approach to monitoring clinical depressive symptoms in social media. In Proceedings of the 2017 IEEE/ACM International Conference on Advances in Social Networks Analysis and Mining 2017, pages 1191-1198.

Ziwei, B. Y. e Chua, H. N. (2019). An application for classifying depression in tweets. In Proceedings of the 2 nd International Conference on Computing and Big Data, ICCBD 2019, page 37-41, New York, NY, USA. Association for Computing Machinery. 